\title{
A case of Mowat-Wilson syndrome with developmental delays and Hirschsprung's disease
}

\author{
Darae Lee ${ }^{1}$, Ja Hye Kim¹, Ja Hyang Cho ${ }^{1}$, Moon-Yun Oh¹, Beom Hee Lee ${ }^{1,2}$, Gu-Hwan Kim², Jin-Ho Choi , and Han-Wook Yoo ${ }^{1,2, *}$ \\ ${ }^{1}$ Department of Pediatrics, ${ }^{2}$ Medical Genetics Center, Asan Medical Center Children's Hospital, University of Ulsan College of Medicine, Seoul, \\ Korea
}

Mowat-Wilson syndrome is an extremely rare genetic disease that is characterized by intellectual disability, facial dysmorphism, Hirschsprung's disease, and other congenital anomalies. This disorder is caused by heterozygous mutations or deletions in the zinc finger E-box-binding homeobox-2 gene (ZEB2). Thus far, approximately 200 cases of Mowat-Wilson syndrome have been reported worldwide. In Korea, only one case with a 2 q22 deletion, which also affects ZEB2, has been previously reported. Here, we describe a patient with Mowat-Wilson syndrome who presented with developmental delays, typical facial dysmorphism, and Hirschsprung's disease. Molecular analysis of ZEB2 identified a novel heterozygous mutation at c.190dup (p.S64Kfs*6). To our knowledge, this is the second report of a Korean patient with Mowat-Wilson syndrome that has been confirmed genetically.

Key words: Mowat-Wilson syndrome, Hirschsprung disease, ZEB2.

\section{Introduction}

Mowat-Wilson syndrome (MWS) is a rare genetic disease that was first described by Mowat and Wilson in 1998 [1]. MWS is characterized by dysmorphic facial features; congenital anomalies such as Hirschsprung's disease, genitourinary anomalies, congenital heart defects, agenesis, or hypogenesis of the corpus callosum; eye defects; and intellectual disability. MWS is caused by mutations or deletions in the zinc finger E-box-binding homeobox-2 gene (ZEB2), and more than 180 different mutations have been identified thus far [2]. Approximately 200 MWS cases have been reported worldwide
[3]; of these, the only report of a Korean patient with MWS was published in 2013 [3]. This patient had a 0.9-Mb deletion at chromosome 2q22.3, which included ZEB2.

Owing to its rarity and broad-spectrum congenital abnormalities, many MWS cases remain undiagnosed. We recently encountered a female MWS patient who presented with global developmental delays, typical facial features of MWS, and Hirschsprung's disease. We believe that our experience with this case could help identify additional cases of MWS, and we suggest that genetic testing could be performed to diagnose syndromic features, including dysmorphism, delayed development, and congenital anomalies.

Received: 31 July 2014, Revised: 10 October 2014, Accepted: 11 October 2014, Published: 31 December 2014

*Corresponding author: Han-Wook Yoo, M.D., Ph.D.

Department of Pediatrics, Asan Medical Center Children's Hospital, University of Ulsan College of Medicine, 88 Olympic-ro 43-gil, Songpa-gu, Seoul 138736, Korea.

Tel: +82-3010-3374, Fax: +82-473-3725, E-mail: hwyoo@amc.seoul.kr

Conflict of interest: We declare that we do not have any conflicts of interests.

(c) This is an open-access article distributed under the terms of the Creative Commons Attribution Non-Commercial License (http://creativecommons.org/licenses/by-nc/3.0/) which permits unrestricted non-commercial use, distribution, and reproduction in any medium, provided the original work is properly cited.

(c) Copyright 2014 by the Korean Society of Medical Genetics 
Case

Our patient was a female born to non-consanguineous parents after 40 weeks of gestation with no perinatal problems. Her birth weight was $2.9 \mathrm{~kg}$ (-0.9 standard deviation score [SDS]). There was no family history of congenital anomalies or intellectual disability. Hirschsprung's disease was diagnosed in the neonatal period. At 4 months of age, the patient underwent a Duhamel operation, and she was able to control her head movements; furthermore, at 8 months, she could roll over. However, at 18 months, she visited the outpatient clinic due to developmental delays. Her height and weight were $79 \mathrm{~cm}(-0.8$ SDS) and $9 \mathrm{~kg}(-1.4 \mathrm{SDS})$, respectively. Her head circumference was $42 \mathrm{~cm}(-3.4$ SDS). She could not sit unaided nor speak any meaningful words. Facial dysmorphism was noted, including hypertelorism, deep-set eyes, epicanthal folds, depressed nasal bridge, and large and posteriorly rotated ears. Cognitive adaptive test/clinical linguistic and auditory milestone scale developmental quotient scores at 14 months were 55.3\% (age equivalent, 7.2 months) and 69.2\% (age equivalent, 9 months), respectively. Echocardiography, abdominal ultrasonography, electroencephalography, brain magnetic resonance imaging, and automated brainstem response audiometry showed no abnormal findings. The patient showed myopia and delayed development visual function upon ophthalmologic evaluation.

Genomic DNA was isolated from the patient's peripheral leukocytes after informed consent was obtained from the parents. Ten exons and the corresponding exon-intron boundaries on ZEB2 were amplified by polymerase chain reaction. Direct sequencing was subsequently carried out using a BigDye v.3.1 terminator kit and ABI3130xl Genetic Analyzer (Applied Biosystems, Foster City, CA, USA). A novel heterozygous frameshift variant, c.190dup(A) (p.S64Kfs*6) in exon 3, was subsequently identified (Fig. 1).

\section{Discussion}

In this report, we describe the second Korean patient who has been diagnosed with MWS and confirmed by molecular analysis. This patient presented with facial dysmorphism, developmental

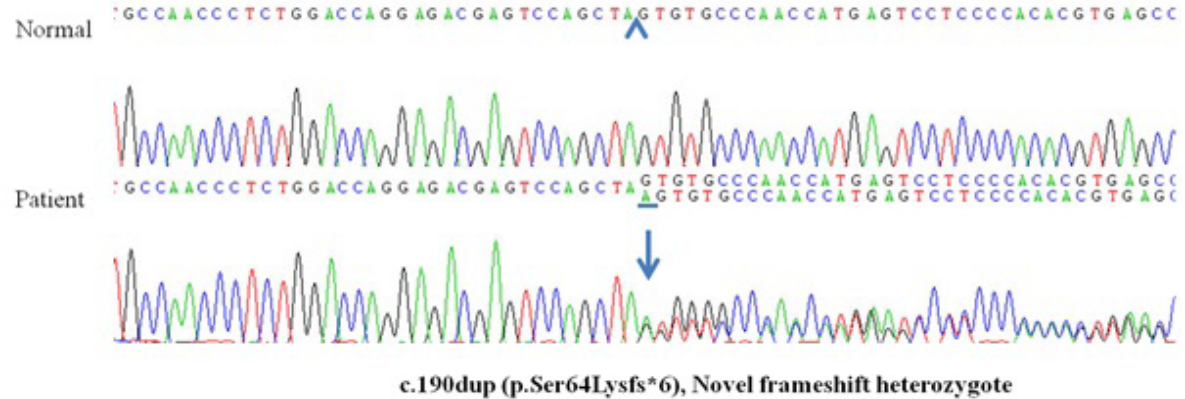

Fig. 1. Partial sequences of $Z E B 2$ in the study patient. A novel frameshift mutation at c.190dup (p.S64Kfs ${ }^{\star} 6$ ) in ZEB2 was identified.

Table 1. Frequencies of clinical findings for Mowat-Wilson syndrome in 192 previously identified patients and the present case [1,5]

\begin{tabular}{|lcc}
\hline & Patients with each clinical feature, $\mathrm{n}(\%)$ & Present case \\
\hline Facial gestalt & $184 / 188(97.87)$ & + \\
\hline Mental retardation & $192 / 192(100)$ & + \\
\hline Microcephaly & $145 / 180(80.56)$ & - \\
\hline Seizures & $116 / 157(73.89)$ & + \\
\hline Hirschsprung's disease & $102 / 187(54.55)$ & - \\
\hline Congenital heart disease & $100 / 185(54.05)$ & - \\
\hline Urogenital/renal anomalies & $86 / 165(52.12)$ & + \\
\hline Short stature & $43 / 85(50.59)$ & - \\
\hline Hypoplasia or agenesis of corpus callosum & $77 / 167(46.11)$ & + \\
\hline Eye anomalies & $7 / 181(3.87)$ & - \\
Cleft palate & $5 / 181(2.76)$ & + \\
\hline
\end{tabular}

Total of reported cases is 192 .

+ , present; -, absent. 
delays, and Hirschsprung's disease. MWS is characterized as a single-gene disorder caused by heterozygous mutation or deletion of ZEB2, which is located on chromosome 2q22. ZEB2 consists of 10 exons containing 1214 amino acids. The product of ZEB2 is the Smad-interacting transcription factor, which also plays various roles in development [4]. In our present case, a novel variant, c. 190dup (p.S64Kfs ${ }^{*}$ ), was identified in $Z E B 2$. This duplication is located on the $\mathrm{N}$-terminal region of $Z E B 2$, resulting in premature termination at residue 70 . This prematurely truncated protein has no zinc finger domain or homeodomain at its $\mathrm{C}$ terminus, both of which are essential for the proper function of ZEB2.

Patients with MWS are initially recognized by various clinical features, including intellectual disability, facial dysmorphism, Hirschsprung's disease, seizures, congenital heart defects, genitourinary anomalies, and eye defects. The frequencies of the clinical manifestations in previously reported cases as well as our present case are summarized in Table $1[1,5]$. The characteristic facial features of MWS include a broad nasal bridge, rounded nasal tip, triangular jaw, broad medial eyebrows, and widely spaced and deep-set eyes, which are noted in most affected patients [1].

Hirschsprung's disease is one of the major manifestations of MWS; this condition is also associated with high mortality in patients with MWS [6]. Therefore, it is important to diagnose Hirschsprung's disease in patients with MWS, especially in those with gastrointestinal symptoms such as constipation and vomiting. In their first description of MWS, Mowat et al. [1] reported that 5 of 6 patients with MWS had Hirschsprung's disease. Bonnard et al. [6] reported that Hirschsprung's disease occurs in almost $50 \%$ of MWS patients. In another study, $70 \%$ of MWS patients developed Hirschsprung's disease as an isolated trait, with other congenital malformations noted in the remaining 30\% of patients, such as congenital heart defects, gastrointestinal malformations, cleft palate, and craniofacial anomalies [7].

It is important to note that Hirschsprung's disease is known to be associated with other syndromic disorders, for example, Shah-Waardenburg syndrome, which is a rare genetic disorder characterized by hypopigmentation of hair, sensorineural hearing loss. In addition, Hirschsprung's disease is occasionally associated with Bardet-Biedl syndrome, Kauffman-McKusick syndrome, Smith-Lemli-Opitz syndrome, and cartilagehair hypoplasia. Among these, Smith-Lemli-Opitz syndrome demonstrates similar clinical features as MWS, such as growth retardation, microcephaly, mental retardation, and hypospadias.
In addition, some patients with Bardet-Biedl syndrome present with eye defects such as pigmentary retinopathy, which can also develop in MWS patients [7]. Hence, careful physical examination for additional features of MWS and prompt genetic testing are needed to confirm any diagnosis.

In patients harboring normal ZEB2 sequences, karyotyping, fluorescent in situ hybridization analysis, multiplex ligationdependent probe amplification analysis, and chromosome microarray analysis can be performed, because some patients may carry chromosomal rearrangements at $2 q 22$ that disrupt ZEB2 ( 2\%) or large genomic deletions at ZEB2 (15\%) [3]. The first Korean MWS patient was diagnosed using high-resolution microarray analysis, which confirmed a 0.9-Mb deletion at 2q22.3 that implicated ZEB2 [3].

Ophthalmological abnormalities have been described in approximately 4\% of MWS patients [8,9], which range from mild features including ptosis, strabismus, and iris/retinal/optic disc colobomas, to more severe features such as optic nerve hypoplasia, retinal atrophy, and aplasia [10]. Epilepsy is one of the major manifestations of MWS. Approximately 70-75\% patients develop seizures at a median age of 14.5 months that are often precipitated by fever [11]. The main seizure types include focal and atypical absence seizures [11]. At the onset of epilepsy, electroencephalography is normal or shows mild slowing of background activities. According to previous reports, epilepsy is refractory to treatment [11]. However, our patient did not develop epileptic episodes until 18 months and demonstrated normal findings on electroencephalography and brain magnetic resonance imaging. Nevertheless, careful monitoring of seizure development is warranted during follow-up evaluations.

Although MWS is characterized by distinct facial features, Hirschsprung's disease, and intellectual disability, this condition is rarely reported because it remains unknown among general practitioners. Our experiences reported herein should help to identify additional MWS cases. Appropriate genetic testing will also provide valuable information for confirming diagnoses, as well as providing genetic counseling to family members.

\section{References}

1. Mowat DR, Croaker GD, Cass DT, Kerr BA, Chaitow J, Adès LC, et al. Hirschsprung disease, microcephaly, mental retardation, and characteristic facial features: delineation of a new syndrome and identification of a locus at chromosome 2q22-q23. J Med Genet 
1998;35:617-23.

2. Stenson PD, Mort M, Ball EV, Shaw K, Phillips A, Cooper DN. The Human Gene Mutation Database: building a comprehensive mutation repository for clinical and molecular genetics, diagnostic testing and personalized genomic medicine. Hum Genet 2014;133:19.

3. Park JY, Cho EH, Lee EH, Kang YS, Jun KR, Hur YJ. Mowat-Wilson syndrome detected by using high resolution microarray. Gene 2013;532:307-9.

4. El-Kasti MM, Wells T, Carter DA. A novel long-range enhancer regulates postnatal expression of Zeb2: implications for MowatWilson syndrome phenotypes. Hum Mol Genet 2012;21:5429-42.

5. Garavelli L, Zollino M, Mainardi PC, Gurrieri F, Rivieri F, Soli F, et al. Mowat-Wilson syndrome: facial phenotype changing with age: study of 19 Italian patients and review of the literature. Am J Med Genet A 2009;149A:417-26.

6. Bonnard A, Zeidan S, Degas V, Viala J, Baumann C, Berrebi D, et al.
Outcomes of Hirschsprung's disease associated with Mowat-Wilson syndrome. J Pediatr Surg 2009;44:587-91.

7. Borrego S, Ruiz-Ferrer M, Fernández RM, Antiñolo G. Hirschsprung's disease as a model of complex genetic etiology. Histol Histopathol 2013;28:1117-36.

8. Garavelli L, Mainardi PC. Mowat-Wilson syndrome. Orphanet J Rare Dis 2007;2:42.

9. Adam MP, Conta J, Bean LH. Mowat-Wilson syndrome. In: Pagon RA, Adam MP, Ardinger HH, Bird TD, Dolan CR, Fong CT, et al., eds. GeneReviews ${ }^{\circledR}$. [http://www.ncbi.nIm.nih.gov.libproxy.amc.seoul. kr:8000/books/NBK1116/]

10. Ariss M, Natan K, Friedman N, Traboulsi El. Ophthalmologic abnormalities in Mowat-Wilson syndrome and a mutation in ZEB2. Ophthalmic Genet 2012;33:159-60.

11. Cordelli DM, Garavelli L, Savasta S, Guerra A, Pellicciari A, Giordano $L$, et al. Epilepsy in Mowat-Wilson syndrome: delineation of the electroclinical phenotype. Am J Med Genet A 2013;161A:273-84. 